\title{
Time Difference of Arrival Passive Positioning Technology and Its Application Research in Table Tennis Ball Landing Spot Estimation
}

\author{
Zhang Jian and Huo Hong
}

\begin{abstract}
Time Difference of Arrival positioning method is typical of passive positioning which has a high positioning accuracy, widely used in the military field. Therefore, this article will apply it to table tennis positioning estimation. The method needs at least three sensors to position two-dimensional target. This paper describes the principle of TDOA and six kinds of classical algorithms for target estimation. Direct Solution Algorithms is selected to estimate table tennis placement after comparing these algorithms. The relationships between positioning accuracy and geometric distribution of sensors are analyzed and simulated in order to optimize the positioning accuracy. Finally, two location schemes are proposed for table tennis placement estimation, and their respective positioning features are analyzed in this paper.
\end{abstract}

Index Terms-Table tennis placement estimation, TDOA, position accuracy.

\section{INTRODUCTION}

Time Difference of Arrival (TDOA) [1] is one of the most important passive location methods. Its location precision is high and without the need of restrict time synchronization. According to the time difference of arrival of acoustic emission sensor array, the emission source location can be estimated by computation [2]. The TDOA technique is introduce to table tennis ball landing spot estimation in this paper.

The objective data deficiency situation can be improved. The erroneous judgement rate can be reduced for the application of the TDOA technique in table tennis. It's helpful for correctness of the trainee capacity evaluation and scientific and reasonable training it is good for stipulating trainee to enhance it capacity further.

With the statistical information of table tennis spot distribution, the coach may discover problems in the process of teaching and training and making targeted improvement, and qualitative and quantitative evaluation of athlete stage training results can be realized. The technology is very important for studying the table tennis rule and can greatly

Manuscript received March 1, 2017; revised April 15, 2017.

Zhang Jian is with the Harbin Normal University, Heilongjiang, China (e-mail: jian9626@sina.com).

Huo Hong is with the Harbin Institute of Technology, China (e-mail: 1044213680@qq.com). improve the combat level of athletes.

\section{PASSive Time DifFerence LoCATION OF ARRIVAL TECHNOLOGY}

Time difference of arrival (TDOA) location method is also called hyperbolic positioning methods, including a number of positioning sensors. Signals radiated from the vibration source can be received by sensors. Vibration source location is estimated through the TDOA of the vibration signal between sensors [3], [4]. According to the practical application TDOA can be divided into 2D plane and 3D space location. In the two-dimensional plane, three sensors are needed at least. As shown in Fig. 1, TDOA of sensor s1 and sensor s2 from vibration source determines a pear of hyperbola whose focuses are of these two sensor positions. In the similar way, another pear of hyperbola is determined by $\mathrm{s} 1$ and $\mathrm{s} 3$. The intersection is the very spot of the vibration source location.

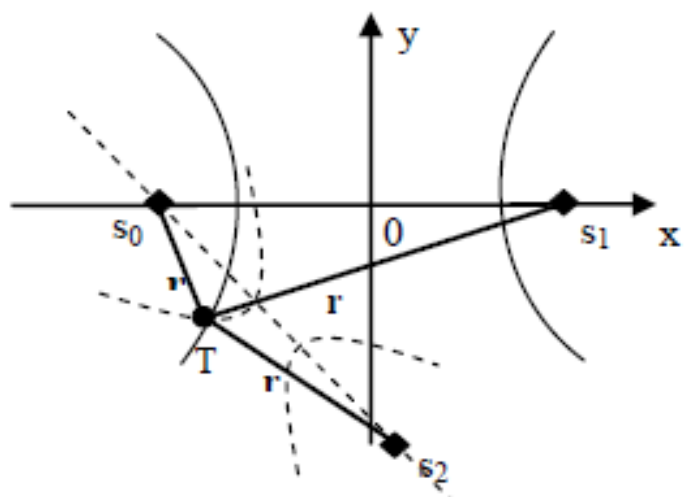

Fig. 1. Schematic of plane TDOA location.

Take three sensor TDOA location system as an example, it is consist of one main sensor and two complement sensors and they are distributed at $\left(x_{j}, y_{j}\right)^{T}, \mathrm{j}=0,1,2 . \mathrm{j}=0$ is for main sensor, $\mathrm{j}=1,2$, is for complement sensors. Vibration source is at $(\mathrm{x}, \mathrm{y})$.

The location function is as below [5]:

$$
\left\{\begin{array}{c}
r_{0}^{2}=\left(x-x_{0}\right)^{2}+\left(y-y_{0}\right)^{2} \\
r_{i}^{2}=\left(x-x_{i}\right)^{2}+\left(y-y_{i}\right)^{2}, i=1,2 \\
\Delta r_{i}=r_{i}-r_{0}=v \cdot \Delta t_{i}, i=1,2
\end{array}\right.
$$

r0: the distance between the vibration source and main sensor 
ri: the distance between the vibration source and complement sensors

$\triangle \mathrm{ri}$ : is the distant difference between vibration source and main sensor.

$\mathrm{v}$ : is propagation velocity of vibration

Function 1 is a non-linear function set. The radiation source location can be estimated by solving the function set. There are a number of algorithms for this function set solving, for example, SX Algorithm, Friedlander Algorithm [6], Fang Algorithm [7], Chan Algorithm [8], Taylor Algorithm [9], Direct Algorithm, so on and so forth.

Through a comparative study and analysis of TDOA technique and 6 solution algorithms, the differences of the algorithms are the complexity in computation, the precision estimation and for different application.

Therefore, the TDOA location method is used to estimate target, should according to the difference of practical application environment to select the most suitable algorithm, for at the lowest price to get the highest precision, at the same time has good reliability and robustness.

\section{Direct Algorithm ACCURACY ANALYSIS AND NUMERICAL SIMULATION}

\section{A. Accuracy Analysis}

Direct algorithm positioning accuracy is derived and analyzed with numerical simulation technology on Matlab. Error equal line is drawn, and the relationship between position error and sensor distribution is given.

Location precision factor is given below:

$$
\begin{aligned}
& G D O P=\sqrt{\sigma_{x}^{2}+\sigma_{y}^{2}} \\
& =\left[\sum_{i=1}^{2} \sum_{j=1}^{2}\left(b_{1 i} b_{1 j}+b_{2 i} b_{2 j}\right) \sigma i_{j}\right]^{-\frac{1}{2}}
\end{aligned}
$$

Equation 2 shows that there are two types of location error in TDOA technology mainly, that is system error and Non Line of sight (NLOS) error. Errors in measuring system mainly include the sensor position error and time difference estimation error. The relative geometric position of sensors has a great influence on the positioning accuracy. Therefore, the relationship between the sensor distribution and Geometric Dilution Precision (GDOP) has to be derived and a reasonable sensor distribution can be proposed.

\section{B. Numerical Simulation}

Based on the above analysis, this section mainly discuss the case when taking standard deviation as a constant, positioning accuracy is discussed the baseline (the straight line between the master sensor and the auxiliary sensor) and the angle formed between baselines impacting on respectively. Therefore, through numerical simulation by MATLAB and the positioning error GDOP curve to be drawn shows the baseline length and angle impacting on positioning accuracy. In order to better observe characteristics of the GDOP positioning error curve, measures is taking in the location error GDOP curve drawing as follows:
Matlab simulation is to create a GDOP 2D matrix. It is the mapping of area to be located. Location accuracy of each spot is calculated. And the fuzzy problem is settled by choosing the smaller value of GDOP. The result is stored in GDOP matrix. The spots with the same accuracy are connected with line and the error value is marked on in order to determine the difference.

The sensor distribution diagram is shown in Fig. 2 in numerical simulation. The main sensor $\mathrm{s}_{0}$ is arranged at the origin of zero, auxiliary sensors $s_{1}$ and $s_{2}$ are arranged on $Y$ axial symmetry. The two baseline intersection angle is $\alpha$. Fig. 3 shows the GDOP with a baseline length of $100 \mathrm{~km}$ and $\alpha=$ $60^{\circ}$.

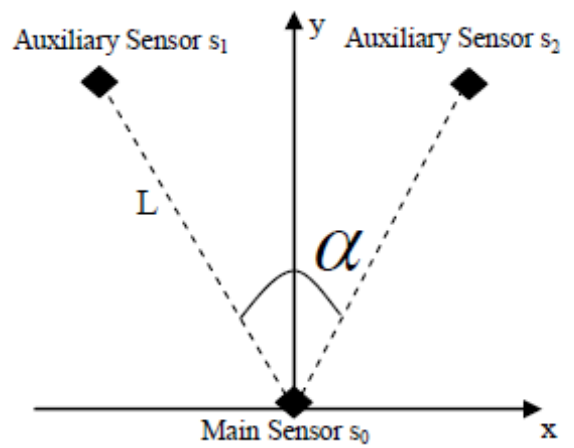

Fig. 2. Sensor distribution diagram.

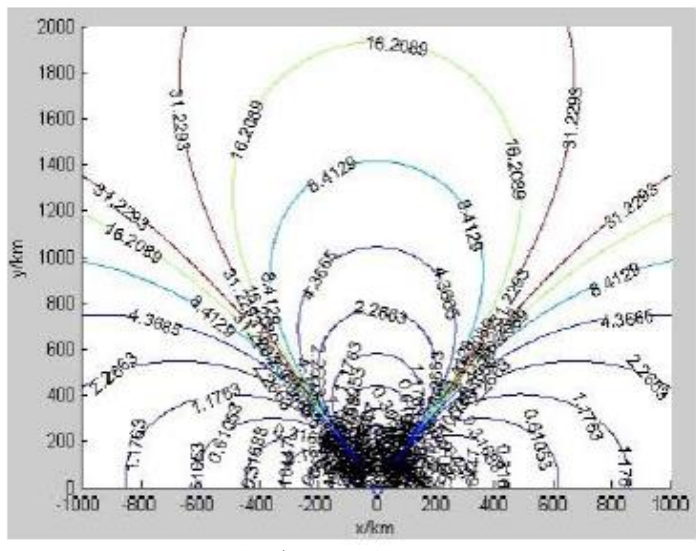

Fig. 3. $\mathrm{L}=100 \mathrm{~km} \alpha=60^{\circ} \mathrm{GDOP}$ curve.

\section{B- I Effect of Baseline Angle on Positioning Accuracy}

As taking the length of baseline as a constant, the relationship between the positioning error and the baseline angle is discussed mainly in this section. The simulation conditions are that the error standard deviation is $20 \mathrm{~ns}$ and the baseline length is $100 \mathrm{~km}$ and the baseline angles are 60 degrees, 90 degrees, 120 degrees and 150 degrees respectively. GDOP error curves are plotted in four cases. Through the above four groups of positioning error curve can be seen:

1) In the zone surrounded by the two baselines, the positioning error is decreased visibly along with $\alpha$ increasing in arithmetic progression, that being from 60 degree to 90 degree, 120 degree till 150 degree. That is that in order to increase positioning accuracy, the baseline intersection angle should be increased.

2) As the distribution of sensor is fixed, the smaller distance the target from the main sensor, the relative error is smaller. The relative error is infinite while the target is on 
baseline.

B-II Effect of Baseline Length on Positioning Accuracy

As taking baseline angle as a constant, the relationship between the positioning error and the baseline length is discussed mainly in this section. The simulation conditions are that the error standard deviation is $20 \mathrm{~ns}$ and the baseline angle is 120 degrees and the baseline length is $100 \mathrm{~km}, 300 \mathrm{~km}$, $500 \mathrm{~km}$ and $700 \mathrm{~km}$ respectively. GDOP error curves are plotted in four cases. Through the above four groups of positioning error curve can be seen:

1) In the zone surrounded by the two base lines, the positioning error is decreased visibly along with $\mathrm{L}$ increasing in arithmetic progression, that being from $100 \mathrm{~km}, 300 \mathrm{~km}, 500 \mathrm{~km}$ and $700 \mathrm{~km}$. That is that in order to improve positioning accuracy, the baseline length should be increased.

2) As the distribution of sensor is fixed, the smaller distance the target from the main sensor, the relative error is smaller. The relative error is infinite while the target is on baseline.

\section{TDOA APPLICATION StUdy ON TABLE TENNIS BALL LANDING SPOT ESTIMATION}

\section{A. Table Tennis Table Stimulation Response Signal Acquisition}

The experimental system structure diagram is shown in Fig. 4. Two non-contact eddy current sensors are arranged as in diagram on a standard tennis table. The stipulating spot is demarcated. Sensor output signals are acquainted via a dual trace oscilloscope. The oscilloscope passes the data to and processed by a computer.

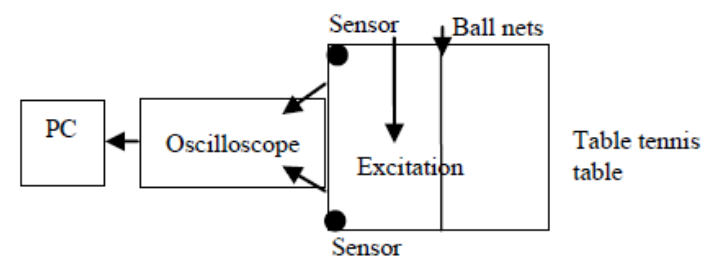

Fig. 4. Block diagram of the experimental system.

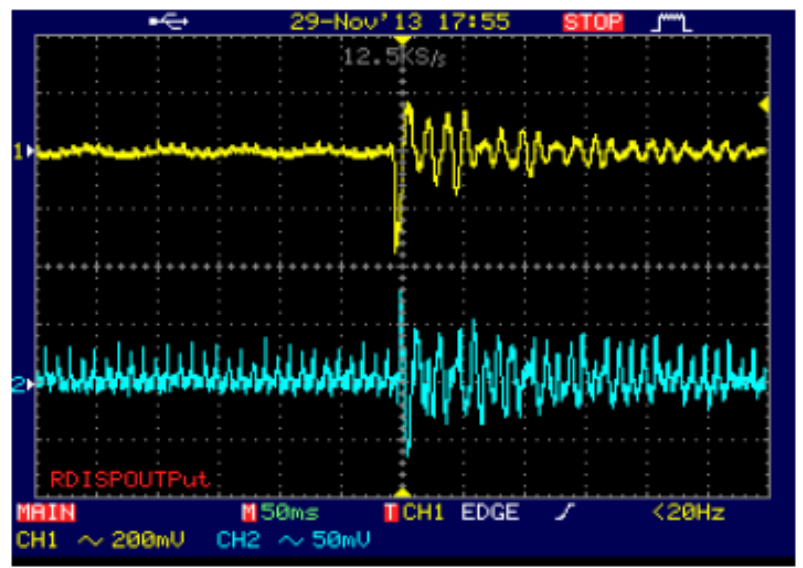

Fig. 5. Sensor output waveform.

Fig. 5 is an example picture of the table response signals collected via two sensors to an excitation. Sampling points are 12500 points. Ten groups of data are collected for each excitation which is excited on the same spot. It includes a group of system noise signals. These data is for off line processing.

\section{B. Sensor Distribution Scheme with Longest Baseline}

Longest baseline of sensor distribution plan is the main sensor is located in the middle position of the net, two auxiliary sensors located in two corners in the lower end of the table tennis table. Its position precision curve is shown in Fig. 6.

Fig. 6 shows of the longest baseline case:

1) The location absolute error is around $15 \sim 43 \mathrm{~mm}$;

2) The location error is smaller in the triangle surrounded with baseline compared with the area outside the triangle;

3) The nearer the target from the main sensor, the smaller the location error is in the triangle zone;

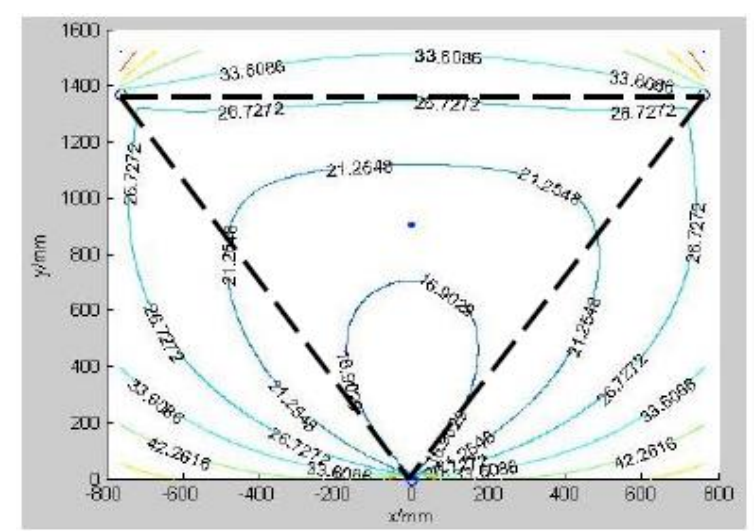

Fig. 6. GDOP curves of the longest baseline case.

\section{Sensor Placement Scheme with Maximum Baseline Angle}

The largest angle of baselines distribution plan is that the main sensor is located in the middle of the net, two auxiliary sensors located at the opposite ends of the net, the angle is 180 degrees. Its position precision curve is shown in Fig. 7.

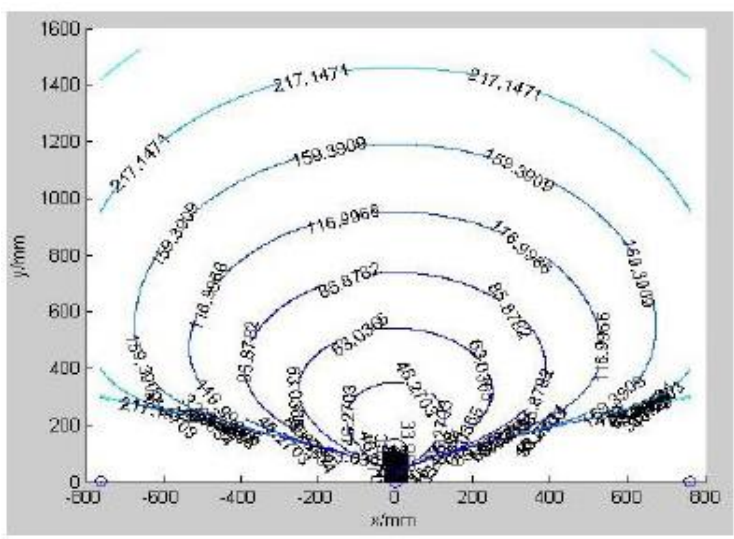

Fig. 7. GDOP curves of maximum angle case.

Fig. 7 shows of the largest intersection angle case:

1) The location absolute error is around $45 \sim 218 \mathrm{~mm}$;

2) In a certain direction, the positioning error is smaller when the distance from the location point is closer to the main sensor. As the distance from the main sensor increases, the positioning error increases.

So the conclusion is:

As in the table tennis application, the location accuracy is much higher in longest baseline case than in the largest 
intersection angle case. And the baseline plays bigger role in location accurate than the angle.

\section{CONCLUSION}

The relationship between TDOA location accuracy and sensor distribution is discussed through theoretical analysis and numerical simulation. Two table tennis sensor distribution schemes are proposed base on the above discussion. The location error curves and its mapping are obtained with Matlab in the longest baseline and the largest intersection angle cases. And the longest baseline scheme is more superior compared with the other scheme in table tennis location application. It is valuable for the design and realization of this kind of system.

\section{REFERENCES}

[1] Y. P. Lei, F. X. Gong, and Y. Q. Ma, "Optimal distribution for for-station TDOA location system," in Proc. International Conference on Biomedical Engineering and Informatics, vol. 3, pp. 2858-2862, March 2010

[2] Z. K. Sun, Y. Y. Zhou, and L. X. He, Single/Multi Bases Initiative/Passive Location Technology, Beijing: National Defense Technology Publishing House, pp. 37-50, 1996.

[3] G. Liu and G. Q. Zhao, "TDOA location and two methods of time difference measurement," Electronic Warfare, pp. 21-25, January 2006.

[4] Z. Y. Hu, B. Yang, and X. W. Li, "Study of wireless location based on hyperbolic type," Information Communications, pp. 18-20, January 2006.
[5] J. M. Delosme, M. Morf, and B. Friedlander, "A linear equation approach to positioning sources from time-difference-of-arrival measurement," in Proc. IEEE Int. Conf. Acoustics, Speech and Signal Processing, pp. 818-824, 1980.

[6] B. Friedlander, "A passive localization algorithm and its accuracy analysis," IEEE Journal of Oceanic engineering, pp. 234-244, December 1987.

[7] M. Aso and E. Al, "A new location estimation method based on maximum likelihood function in cellular system," IEEE VTC, pp. 106-110, 2001.

[8] W. H. Foy, "Position location solutions by taylor series estimation," IEEE Trans on Aerospace and Electronic Systems, vol. AES-12, 1976

[9] J. Geng, "Study of location algorithm of cellular system," Nanjing Post and Communications University, 2012.

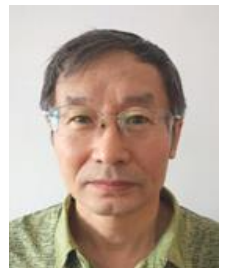

Zhang Jian was born on November 7, 1960, Harbin, China. $\mathrm{He}$ is major in precision instrument and graduated in 1983. He got the bachelor in electromagnetism measuring instrumentation in 1987 the master in test and measurement technology and equipment in 2007; and the doctor degree from Harbin University of Science and Technology. He is study in test and measurement technology and instrumentation, location estimation technology and the instrumentation for the aged based on internet of things. He published EI papers are more than ten, and more than ten China Invention Patents.

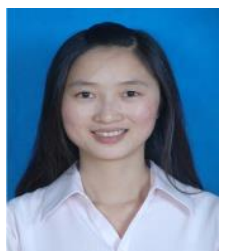

Huo Hong was born on January 27, 1989. She received a master's degree from Harbin Normal University. Now she is studying in Harbin Institute of Technology. The research field is the test measurement technology and instruments. 